\title{
An Autopsied Case of Malignant Sarcomatoid Pleural Mesothelioma in Which Chest Pain Developed Several Months Earlier without Abnormality on Imaging
}

\author{
Daizo Yaguchi $^{a}$ Motoshi Ichikawa $^{a}$ Noriko Inoue $^{a}$ Daisuke Kobayashi ${ }^{\mathrm{a}}$ \\ Akinobu Matsuura $^{a}$ Masato Shizu $^{a}$ Naoyuki Imai ${ }^{a}$ Kazuko Watanabe ${ }^{b}$ \\ Departments of ${ }^{a}$ Respiratory Medicine and ${ }^{b}$ Pathology, Gifu Prefectural Tajimi Hospital, \\ Tajimi, Japan
}

\section{Key Words}

Malignant pleural mesothelioma Sarcomatoid pleural mesothelioma $\cdot$ Chest pain

\begin{abstract}
The patient experienced chest pain for about 7 months, but a diagnosis could not be made until after death. He was diagnosed with malignant sarcomatoid pleural mesothelioma on autopsy. In this case report, difficult aspects of the diagnosis are discussed. The 70-year-old Japanese man was a driver who transported ceramic-related products. Right chest pain developed in July 2013, but no abnormality was detected on a chest computed tomography (CT) performed in September 2013, and the pain was managed as right intercostal neuralgia. A chest CT performed in late October 2013 revealed a right pleural effusion, and the patient was referred to our hospital in early November 2013. Thoracentesis was performed, but the cytology was negative, and no diagnosis could be made. Close examination was postponed because the patient developed a subarachnoid hemorrhage. He underwent ${ }^{18} \mathrm{~F}-$ fluorodeoxyglucose positron emission tomography $\left({ }^{18} \mathrm{~F}-\mathrm{FDG} \mathrm{PET}\right)$ after discharge from the neurosurgery department, and extensive right pleural thickening and ${ }^{18} \mathrm{~F}-\mathrm{FDG}$ accumulation in this region were observed. Based on these findings, malignant pleural mesothelioma was suspected, and a thoracoscopy was performed under local anesthesia in early December 2013, but no definite diagnosis could be made. The patient selected best supportive care and died about 7 months after the initial development of right chest pain. The disease was definitively diagnosed as malignant sarcomatoid pleural mesothelioma by a pathological autopsy.
\end{abstract}

KARGER 125:s $\quad \begin{aligned} & \text { Daizo Yaguchi } \\ & \text { Department of Respiratory Medicine } \\ & \text { Gifu Prefectural Tajimi Hospital } \\ & \text { 5-161, Maehata-cho, Tajimi, Gifu 507-8522 (Japan) } \\ & \text { E-Mail yaguzo0414@gmail.com }\end{aligned}$


Yaguchi et al.: An Autopsied Case of Malignant Sarcomatoid Pleural Mesothelioma in Which Chest Pain Developed Several Months Earlier without Abnormality on Imaging

When chronic chest pain of unknown cause is observed and past exposure to asbestos is suspected, actions to prevent delay in diagnosis should be taken, including testing for suspicion of malignant pleural mesothelioma.

(C) 2015 The Author(s)

Published by S. Karger AG, Basel

\section{Introduction}

Malignant pleural mesothelioma (MPM) was once a rare finding, but patients with asbestos-related mesothelioma have increased in numbers over the last few decades [1, 2]. A pathological diagnosis of MPM cannot be made by close medical examination in some cases, and it is one of the diseases that are difficult to diagnose before death. Symptoms may increase while the disease cannot be definitely diagnosed. For this disease with a poor prognosis, early definitive diagnosis and initiation of treatment are important.

\section{Case Report}

The patient, a 70-year-old Japanese man, was a driver who transported ceramic-related products (asbestos exposure was unclear). The patient became aware of right chest pain in July 2013 and visited a hospital. A chest computed tomography (CT) was performed, but the cause was unclear, and analgesics were prescribed. He visited several medical institutions, but the cause of his pain was not determined, and the pain was managed as right intercostal neuralgia. Since his right chest pain did not improve, he visited the pain clinic of our hospital in mid-September 2013. The pain persisted, and examination at a university hospital pain clinic was scheduled. When the patient was re-examined by a chest CT in another hospital in late October 2013, a right pleural effusion was observed, for which the patient was referred to our hospital in early November 2013. He developed a subarachnoid hemorrhage not accompanied by an aneurysm several days after visiting our hospital and was immediately admitted to our hospital. The patient's medical history was herpes zoster at 62 years of age, surgery for right inguinal hernia at 70 years of age, and he did not smoke.

Status on Referral Visit. The patient's status was as follows: blood pressure, 140/72 mm $\mathrm{Hg}$; pulse, 76/min; $\mathrm{SpO}_{2}$ (under room air inhalation), 98\%; no anemia or jaundice, and no superficial lymph node palpated. On chest auscultation, his right breath sound was weak. No abnormality was noted in the abdominal or reproductive organs.

Blood and Pleural Effusion Test Findings. There were no unusual findings in either the blood cell counts or biochemistry. The serum tumor markers were normal, and the soluble mesothelin-related peptide level was within the normal range at 0.8. In the pleural effusion, the hyaluronic acid level was $27,600 \mathrm{ng} / \mathrm{ml}$, carcinoembryonic antigen (CEA) was $0.5 \mathrm{ng} / \mathrm{ml}$, and cytokeratin fragment was $55.0 \mathrm{ng} / \mathrm{ml}$. An examination of the pleural effusion obtained by right thoracentesis showed a yellow and turbid liquid. The total protein level was 5.2 $\mathrm{g} / \mathrm{dl}$, and the lactate dehydrogenase was $376 \mathrm{IU} / \mathrm{l}$, showing an exudative pattern, and the lymphocyte rate was $63 \%$ on cell fractionation. No atypical cells were noted, and the acidfast bacterial test was negative.

Plain Chest Radiography Findings. A right pleural effusion was noted.

Chest CT Findings (fig. 1). No pleural thickening or pleural effusion was observed on CT in September 2013, but a right pleural effusion accompanied by right pleural thickening was noted in late October 2013. No apparent pleural plaque was detected. 
Yaguchi et al.: An Autopsied Case of Malignant Sarcomatoid Pleural Mesothelioma in Which Chest Pain Developed Several Months Earlier without Abnormality on Imaging

${ }^{18}$ F-Fluorodeoxyglucose Positron Emission Tomography/CT Findings (fig. 2a). 18Ffluorodeoxyglucose positron emission tomography (18F-FDG PET)/CT was performed in early December 2013. Multiple 18F-FDG PETs were performed in the region corresponding to the thickening of the right pleura, and the maximum standardized uptake value $\left(S_{U} V_{\text {max }}\right)$ was 7.7.

Thoracoscopy under Local Anesthesia (fig. 2b). Thoracoscopy was performed in early December 2013. Pleural plaques were present in the parietal pleura, and many small white protrusions, pleural redness, and distension of the blood vessels were observed. A pleural biopsy from the protrusions was performed, and a chest drain was placed in the patient.

Pathological Findings of the Pleural Biopsy. Outgrowth of cells containing oval nuclei with slightly dense chromatin or of spindle cells was noted in fibrous connective tissue accompanied by severe hyaline degeneration. MPM, sarcomatoid pulmonary adenocarcinoma, and fibrous pleurisy were examined for differentiation. Immunostaining was performed, but it was unclear whether the lesion was lung cancer or mesothelioma due to the presence of fibrous pleurisy in the background, and thus, a definite diagnosis could not be made.

Pleural biopsy under general anesthesia was inapplicable because a subarachnoid hemorrhage had developed. The patient and his family did not request CT-guided pleural biopsy on consultation, and best supportive care was selected. The systemic condition gradually worsened, and the patient died in February 2014, 7 months after the development of chest pain and 3 months after the referral visit to our hospital.

Macroscopic Findings (fig. 3a). The whole body was autopsied excluding the brain. In the right lung, marked pleural hypertrophy was observed; a tumor had extended along the pleura while forming nodes at several sites, and the pleura partially adhered to the costal bone. There was no lesion in the right lung parenchyma, left pleura, or abdominal cavity, but metastases were noted in the epicardium, liver, skin, and hilar lymph node.

Histologically, severe fibrosis of the pleura accompanied by hyaline degeneration was noted, and markedly atypical tumor cells containing spindle or multiform nuclei with small nucleoli had infiltrated the pleura and proliferated. On immunostaining of these tumor cells, AE1/AE3 was positive; cytokeratin 7 was partially positive; vimentin was partially positive; calretinin was partially positive; HBME1 was negative; WT1 was negative; CEA was negative; Ber-EP4 was negative, and tissue-specific transcription factor 1 was negative. Based on the immunostaining and hematoxylin-eosin staining (fig. 3b-e), malignant sarcomatoid pleural mesothelioma was definitively diagnosed. We did not look for asbestos in the autopsied lung.

\section{Discussion}

MPM is a rare disease accounting for $1 \%$ of the cases of lung cancer and malignant tumors arising from the lung, and the incidence is 1-2 per million people. Its association with asbestos exposure was reported after it was initially reported by Wagner et al. [1], and the incidence has recently increased worldwide. Our patient was diagnosed with asbestos exposure-induced MPM because pleural plaques were observed on autopsy. According to the Annual of the Pathological Autopsy Cases and database of the Japanese Society of Pathology, 70 and 92 patients with MPM were autopsied in 2011 and 2012, respectively. The incidence in Japan is closely related to mass exposure to asbestos during the high economic growth period, and the import volume of asbestos was high in the 1990s. Assuming that the mean time to mesothelioma development after exposure to asbestos is about $30-40$ years, the incidence is expected to peak in about 2030, so the number of patients will increase in the 
Yaguchi et al.: An Autopsied Case of Malignant Sarcomatoid Pleural Mesothelioma in Which Chest Pain Developed Several Months Earlier without Abnormality on Imaging

future. The prognosis is very poor. The median survival time without treatment is estimated to be 7-8 months, and no standard treatment for advanced cases has been established.

The initial symptoms of MPM are generally chest pain due to pleural infiltration and shortness of breath due to pleural effusion. About $80 \%$ of the cases were accompanied by pleural effusion at the time of discovery, but early lesions show only pleural effusion, and tumor-related pleural thickening may not be observed on imaging, which is important for physicians to know. Mesothelioma arises as a granular tumor of the parietal pleura. The tumor is localized in the parietal pleura, and no tumor is observed in the visceral pleura at this stage. Asymptomatic pleural effusion is the only clinical finding in many cases, and most cases are discovered at a health checkup. The feature of this stage is a localized tumor in the parietal pleura with no tumor in the visceral pleura. This tumor mainly infiltrates locally into the chest wall with a few cases of distant metastasis [3]. Accordingly, the chest pain observed in MPM is due to local chest wall infiltration, not pleural chest pain. Intercostal nerve infiltration-induced intercostal neuralgia is assumed to be included in this. The chest pain experienced by our patient may have been due to intercostal neuralgia accompanying the local chest wall infiltration of the tumor arising from the parietal pleura at a level undetectable by CT because no abnormality was observed on CT 2 months after the development of chest pain. The discovery of malignant diseases in outpatients at pain clinics has been occasionally reported. Our patient had also been treated at the pain clinic of the Anesthesiology Department of our hospital before the development of the pleural effusion, at which time it was considered as pain after herpes zoster, and an intercostal nerve block was proposed. He was going to visit a university hospital pain clinic. Patients with intractable pain are often referred to pain clinics by other hospitals or departments, and the discovery of malignant diseases in such cases has been occasionally reported [4]. It is necessary to pay attention to the presence or absence of malignant diseases in patients who are referred to pain clinics for chest pain of unknown cause. To our knowledge, there has been no previous case in which MPM-induced chest pain developed several months earlier than the observation of pleural effusion and pleural thickening on imaging. Thus, the present case may be valuable in warning physicians to consider malignant disease when chest pain occurs and the CT does not show a lesion.

It is difficult to definitely diagnose MPM based on cytology of the pleural effusion and pleural biopsy, and it may not be possible to diagnose this cancer before death. The rate of diagnosis by pleural effusion cytology was reported to be $26 \%$ [5], and the positive rate is lower in the sarcomatoid than in the epithelioid type [6]. The rates of diagnosis by blind percutaneous pleural biopsy and CT-guided pleural biopsy were reported to be 31\% [7] and $80 \%$ [8], respectively. Regarding invasive diagnostic methods, thoracoscopy under local anesthesia is considered important. The disease was definitively diagnosed by thoracoscopy under local anesthesia in 18 of 20 patients (90\%) in a study involving 64 patients reported by Yamamoto et al. [9] and in 17 of 18 patients (94\%) in a study reported by Muzaffer et al. [8]. However, the diagnosis rate varies among reports, and it is considered that $30-40 \%$ of the cases are difficult to diagnose even by thoracoscopy under local anesthesia [10]. Moreover, sampling of tumor tissue may not be possible even though many biopsies are performed. Takabe et al. [10] presented multiple protrusion and nonspecific hypertrophy types as macroscopic characteristics of MPM based on thoracoscopic findings, and they reported that infiltration of tumor cells was noted in the superficial layer of the mesothelium in biopsy specimens in all cases of the protrusion type, whereas tumor cells were observed under the mesothelium, and the superficial layer was covered with less atypical mesothelial cells in the hypertrophy type. The protrusion type could be easily diagnosed by thoracoscopic biopsy, but the hypertrophy type was difficult to diagnose. In our patient, the lesion had protrusions 
Yaguchi et al.: An Autopsied Case of Malignant Sarcomatoid Pleural Mesothelioma in Which Chest Pain Developed Several Months Earlier without Abnormality on Imaging

on thoracoscopy, but it was classified as the hypertrophy type. Therefore, a biopsy of the deep layer is necessary, including collecting a specimen by cutting the superficial layer of the parietal pleura, because only nonspecific findings were observed on pleural biopsy. In addition, the region with marked $18 \mathrm{~F}-\mathrm{FDG}$ accumulation on PET/CT was located on the mediastinal side, and the thoracoscopic biopsy was inapplicable to this region, indicating another reason for the difficulty in diagnosis. To increase the diagnostic rate, biopsy by round resection of the pleura to the deep layer is performed using a high-frequency knife in cases of pleural thickening from which a sufficient amount of specimen cannot be collected using the standard biopsy forceps [11], or tissue biopsy targeting the region with ${ }^{18}$ F-FDG accumulation is performed. We also performed a tissue biopsy targeting the region with ${ }^{18 F-F D G ~ a c-~}$ cumulation on PET/CT in the patient, but the collected tumor tissue was insufficient and could not be definitively diagnosed.

The diagnostic accuracy of MPM based on 18F-FDG accumulation on PET/CT is high, and favorable ${ }^{18}$ F-FDG accumulation is observed in many cases. However, pleural lesions physically move with respiration and are influenced by heartbeat, and the accumulation may appear pale. Flores et al. [12] reported that stage 1a early cancer could not be detected by ${ }^{18} \mathrm{~F}$ FDG PET, and false-negative cases have also been reported [13]. Thus, physicians should pay attention to the fact that accumulation on PET/CT may not be observed in some cases. In our patient, a pale accumulation in the pleura may have been detected on PET/CT even though no finding was observed on CT, but CT may still be the first choice for screening small pleural lesions because it is superior in spatial resolution.

MPM mainly infiltrates the local region in typical cases, and brain metastasis is rare. In a study on multiple organ metastasis of MPM in 172 autopsied cases, the frequency of metastasis decreased in the order of the liver, adrenal gland, kidney, and contralateral side of the lung, and brain metastasis was observed in only $3 \%$ of the cases [14]. In most patients with brain metastasis of MPM, MPM was diagnosed on autopsy after death. When the frequency of metastasis was compared between the histologic types, the frequency was significantly higher in the sarcomatoid type than in the biphasic and epithelioid types [15]. Our patient concomitantly developed a subarachnoid hemorrhage. The presence or absence of brain metastasis was unclear because the brain was not autopsied. Although the possibility of brain metastasis-induced subarachnoid hemorrhage cannot be ruled out, since the subarachnoid hemorrhage was not accompanied by an aneurysm and no finding was observed on magnetic resonance imaging, we thought that the possibility of brain metastasis was low. In addition, to our knowledge, there has been no report connecting MPM to subarachnoid hemorrhage. However, to clarify the causal relationship, we should have autopsied the brain in this patient.

\section{Conclusion}

When asbestos exposure is suspected in a patient with chronic chest pain, it is important to take MPM into consideration in treatment even though no abnormality is observed on CT.

\section{Acknowledgments}

The authors would like to thank all doctors at the Department of Respiratory Medicine and Pathology. 


\section{Case Reports in Oncology}

\begin{tabular}{l|l}
\hline \multicolumn{2}{l}{ Case Rep Oncol 2015;8:439-446 } \\
\hline DOI: 10.1159/000441468 & $\begin{array}{l}\text { (C) 2015 The Author(s). Published by S. Karger AG, Basel } \\
\text { www.karger.com/cro }\end{array}$ \\
\hline
\end{tabular}

Yaguchi et al.: An Autopsied Case of Malignant Sarcomatoid Pleural Mesothelioma in Which Chest Pain Developed Several Months Earlier without Abnormality on Imaging

\section{Statement of Ethics}

Informed consent was obtained from the patient's family for publication of this case report and any accompanying images.

\section{Disclosure Statement}

The authors declare that they have no competing interests.

\section{References}

1 Wagner JC, Sleggs CA, Marchand P: Diffuse pleural mesothelioma and asbestos exposure in the North Western Cape Province. Br J Ind Med 1960;17:260-271.

2 Kisihimoto T, Ozaki S, Kato K, et al: Malignant pleural mesothelioma in parts of Japan in relationship to asbestos exposure. Ind Health 2004;42:435-439.

3 Brenner J, Sordillo PP, Magill GB, et al: Malignant mesothelioma of the pleura: review of 123 patients. Cancer 1982;49:2431-2435.

4 Sakuramoto C, Koura M, Koiso S, et al: Two cases of severe low back pain finally diagnosed as multiple spine metastasis of malignant tumor. JJSPC 2006;13:122-127.

5 Boutin C, Rey F: Thoracoscopy in malignant mesothelioma: a prospective study of 188 consecutive patients; part 1. Diagnosis. Cancer 1993;72:389-404.

6 Tao LC: The cytopathology of mesothelioma. Acta Cytol 1979;23:209-213.

7 Biswagit C, Ida R, John S, et al: The role of Abrams percutaneous pleural biopsy in the investigation of exudative pleural effusions. Chest 2006;129:1549-1555.

-8 Muzaffer M, Guntulu A, Emine D, et al: Medical thoracoscopy vs CT scan-guided Abrams pleural needle biopsy for diagnosis of patients with pleural effusions. Chest 2010;137:1362-1368.

-9 Yamamoto T, Moroboshi T, Nagashima T: Clinical diagnosis of malignant pleural mesothelioma. Jap J Thorac Surg 2007;60:10-13.

10 Takabe K, Shimizu T, Tsukada Y, et al: Thoracoscopic appearance and histological findings of nine cases of pleural malignant mesothelioma in relation to clinical findings. JJLC 1997;37:335-345.

11 Sasada S: Semiflexible thoracoscopy under local anesthesia. JJSRE 2008;30:293-299.

12 Flores RM, Akhurst T, Gonen M, et al: Positron emission tomography defines metastatic disease but not locoregional disease in patients with malignant pleural mesothelioma. J Thorac Cardiovasc Surg 2003;126:11-16.

-13 Sørensen JB, Ravn J, Loft A, et al: Preoperative staging of mesothelioma by ${ }^{18 F-f l u o r o-2-d e o x y-D-g l u c o s e ~}$ positron emission tomography/computed tomography fused imaging and mediastinoscopy compared to pathological findings after extrapleural pneumonectomy. Eur J Cardiothorac Surg 2008;34:1090-1096.

14 Kobayashi T, Tsugawa T, Hashizume C, et al: Gamma knife radiosurgery of brain metastasis from malignant pleural mesothelioma - report of three cases with autopsy study in a case. Nagoya J Med Sci 2014;76:187193.

15 Law MR, Hodoson ME, Heard B, et al: Malignant mesothelioma of the pleura: relation between histological type and clinical behavior. Thorax 1982;37:810-815. 


\section{Case Reports in Oncology}

\begin{tabular}{l|l}
\hline Case Rep Oncol 2015;8:439-446 \\
\hline DOI: 10.1159/000441468 & $\begin{array}{l}\text { (c) 2015 The Author(s). Published by S. Karger AG, Basel } \\
\text { www.karger.com/cro }\end{array}$ \\
\hline
\end{tabular}

Yaguchi et al.: An Autopsied Case of Malignant Sarcomatoid Pleural Mesothelioma in Which Chest Pain Developed Several Months Earlier without Abnormality on Imaging
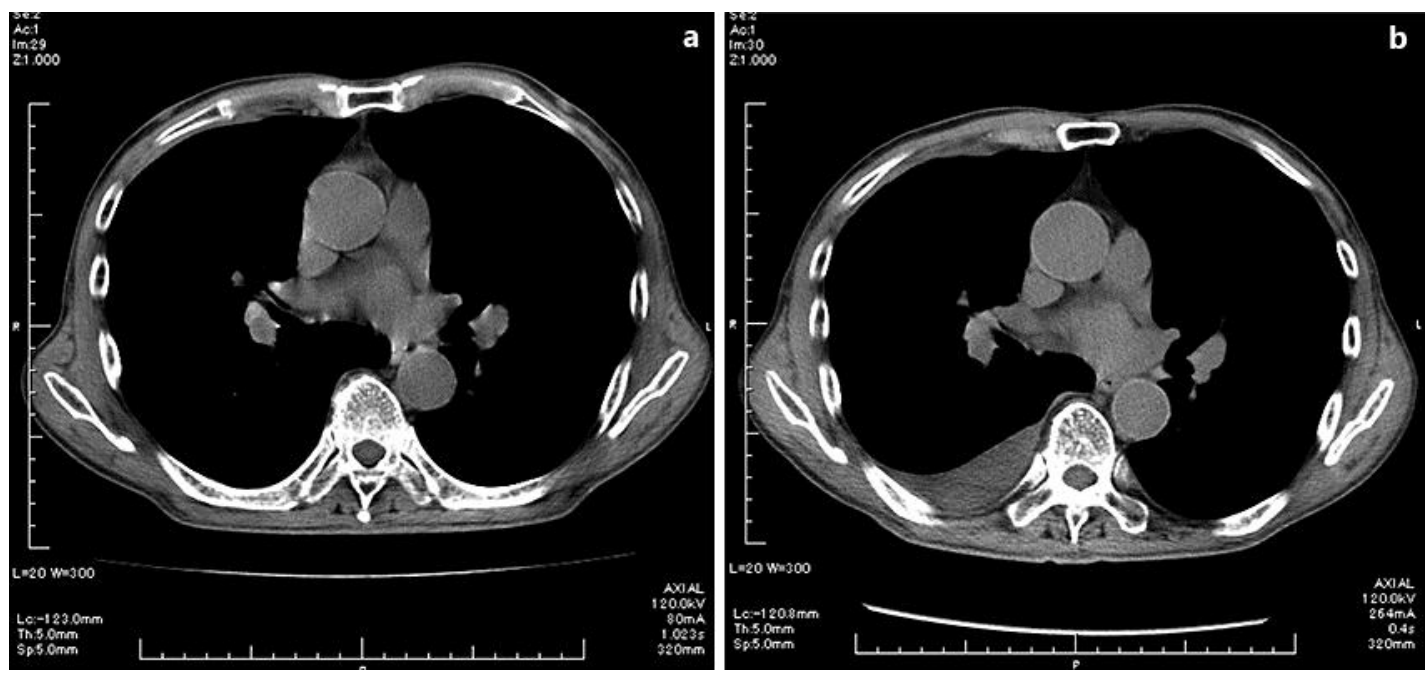

Fig. 1. a No pleural thickening, pleural effusions, or pleural plaques were observed about 2 months after the development of right chest pain. $\mathbf{b}$ Right pleural thickening accompanied by right pleural effusion was observed about 4 months after the development of right chest pain.
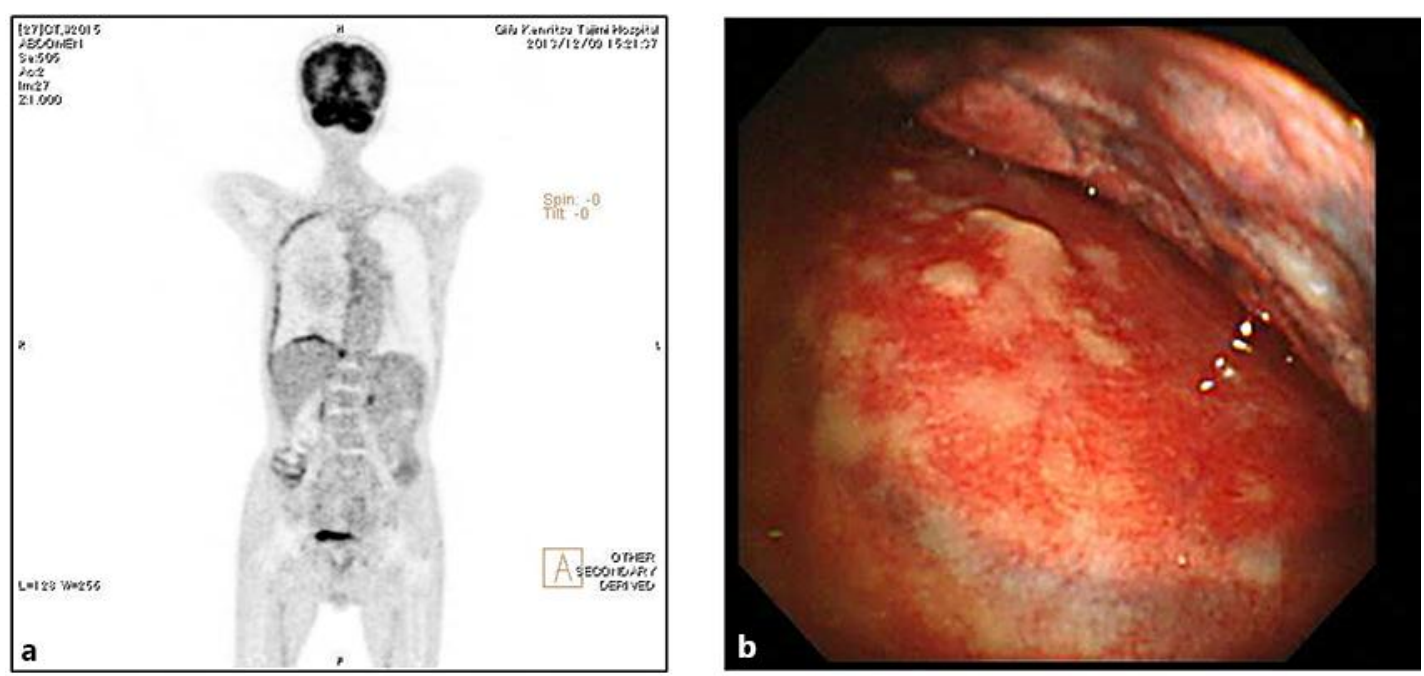

Fig. 2. a An ${ }^{18}$ F-FDG PET/CT scan showed ${ }^{18}$ F-FDG accumulation on the thickened pleura. b A thoracoscopic examination under local anesthesia shows the internal view of the thorax with multiple irregular nodules on the parietal pleura. Vessels are markedly distinct around the white nodules. 


\section{Case Reports in Oncology}

\begin{tabular}{l|l}
\hline \multicolumn{2}{l}{ Case Rep Oncol 2015;8:439-446 } \\
\hline DOI: $10.1159 / 000441468$ & $\begin{array}{l}\text { (c) 2015 The Author(s). Published by S. Karger AG, Basel } \\
\text { www.karger.com/cro }\end{array}$ \\
\hline
\end{tabular}

Yaguchi et al.: An Autopsied Case of Malignant Sarcomatoid Pleural Mesothelioma in Which Chest Pain Developed Several Months Earlier without Abnormality on Imaging
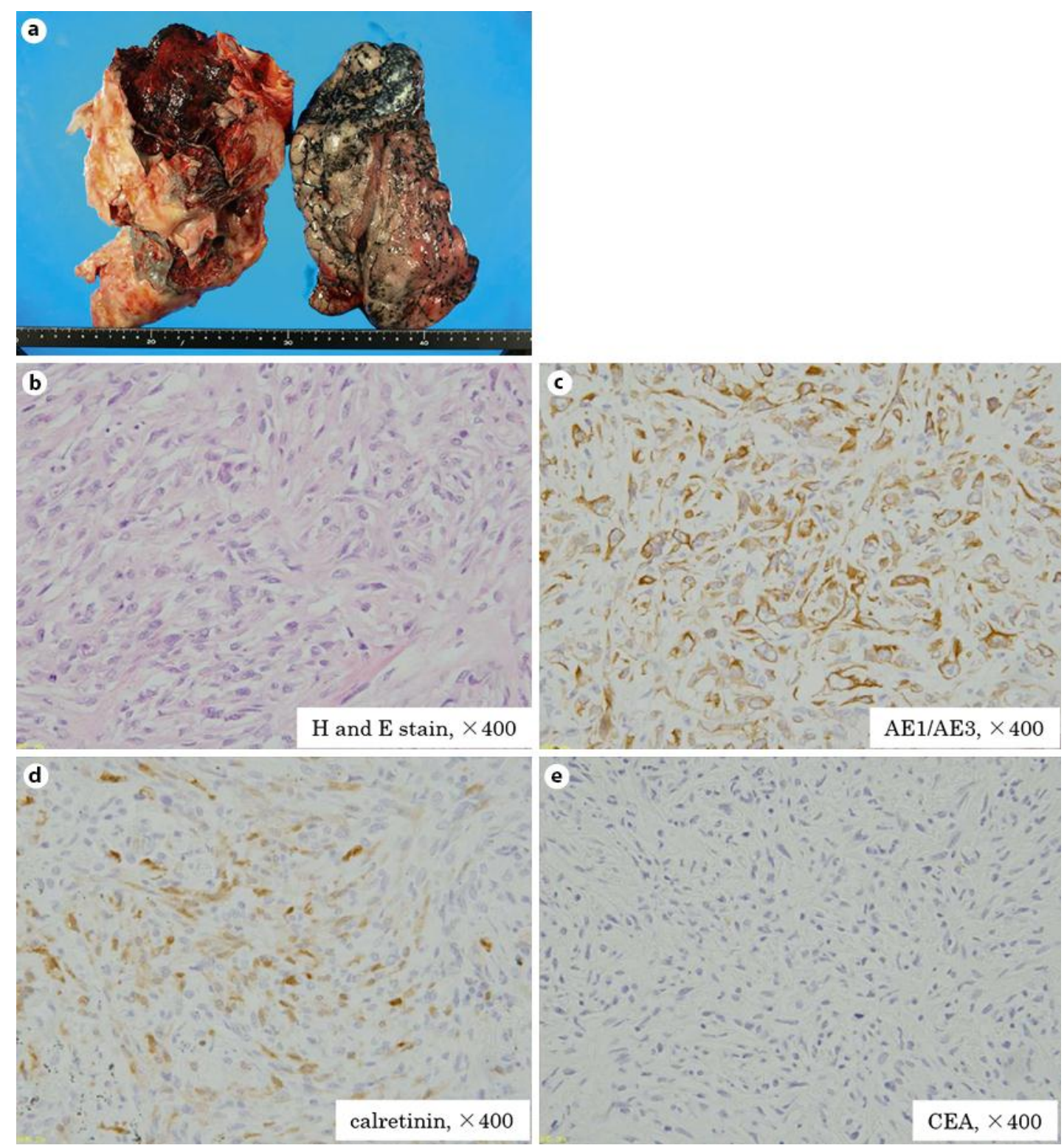

Fig. 3. Macroscopic appearance of the right lung autopsy specimens showing marked pleural thickening and tumors extending along the pleural surface (a). Markedly atypical tumor cells, containing spindle or multiform nuclei with small nucleoli, had infiltrated the pleura and proliferated (b). Tumor cells stained strongly positive for AE1/AE3 (c) and calretinin (d) and stained negative for CEA (e). 